\section{Case Reports in Ophthalmology}

Case Rep Ophthalmol 2018;9:114-118

DOI: $10.1159 / 000485965$

Published online: February 16, 2018
(C) 2018 The Author(s)

Published by S. Karger AG, Basel www.karger.com/cop

\title{
A Case of Neurotrophic Keratopathy Associated with Nasopharyngeal Carcinoma
}

\author{
Tomoko Sato Koji Sugioka Aya Kodama-Takahashi Masahiko Fukuda \\ Hiroshi Mishima Yoshikazu Shimomura \\ Department of Ophthalmology, Kindai University Faculty of Medicine, \\ Osaka-Sayama, Japan
}

\section{Keywords}

Neurotrophic keratopathy $\cdot$ Nasopharyngeal carcinoma $\cdot$ Cavernous sinus

\begin{abstract}
Purpose: To report a case of neurotrophic keratopathy associated with nasopharyngeal carcinoma. Case Report: A 59-year-old man who had been diagnosed with a nasopharyngeal carcinoma was referred to the authors because of visual disturbance and pain in his right eye. Slit-lamp examination revealed a corneal epithelial defect and corneal stromal edema surrounding the epithelial defect area in his right eye. Magnetic resonance imaging showed a mass in his cavernous sinus, which was identified as nasopharyngeal carcinoma (NPC). We diagnosed neurotrophic keratopathy associated with NPC and initiated treatment with preservative-free artificial tears, antibiotic eye drops, fibronectin, a therapeutic contact lens, and amniotic membrane transplantation. However, the persistent corneal epithelial defect was unresponsive to these treatments. Conclusion: Neurotrophic keratopathy secondary to NPC is thought to be rare. We presented a case of neurotrophic keratopathy associated with cavernous sinus metastasis of an NPC. The development of new and more effective treatments for this refractory disease is anticipated.

(C) 2018 The Author(s)

Published by S. Karger AG, Basel
\end{abstract}




\section{Case Reports in Ophthalmology}

\section{Case Report}

A 59-year-old man was referred to the authors in July 2016 because of visual disturbance and pain in his right eye. He had been diagnosed with a nasopharyngeal carcinoma (NPC) and had undergone chemotherapy and radiotherapy over a course of 60 months. He presented with diplopia that had developed over 28 months. His best-corrected visual acuity was 20/200 OD and 20/20 OS. Intraocular pressure was normal in both eyes. Slit-lamp examination and fluorescein staining revealed a corneal epithelial defect and corneal stromal edema surrounding the epithelial defected area in his right eye (Fig. 1). On the other hand, no pathological changes were observed in his left cornea. Corneal sensitivity was measured using a Cochet-Bonnet esthesiometer. Central corneal sensitivity was very low in his right eye $(<5 \mathrm{~mm})$ and normal in his left eye $(50 \mathrm{~mm})$. Tear secretion was normal in both eyes. Examination with a confocal microscope (Heidelberg Retina Tomograph II Rostock Cornea Module; Heidelberg Engineering, Heidelberg, Germany) revealed that the subbasal nerves were visible in the left cornea but not visible in the right cornea (Fig. 2). On external examination, there was no evidence of ptosis. Upon further examination, no pathological changes were detected in the lens, vitreous humor, fundus, and optic nerve. Magnetic resonance imaging (MRI) showed a mass in his cavernous sinus, which was identified as an NPC (Fig. 3). The soft tissue tumor occupied the right Meckel's cave (Fig. 3b). The trigeminal ganglion is located within the Meckel's cave. Persistent corneal epithelial defect did not improve over 2 weeks.

Based on the corneal manifestations, loss of corneal sensitivity, and MRI findings, we diagnosed neurotrophic keratopathy associated with NPC. Subsequently, the patient was prescribed a 60-month course of chemotherapy and radiotherapy, and we initiated treatment with preservative-free artificial tears and antibiotic eye drops. The persistent corneal epithelial defect was unresponsive to artificial tears, fibronectin, and a therapeutic contact lens. The corneal epithelial defect did not completely heal in 4 months. Finally, because amniotic membrane transplantation is an effective treatment for refractory neurotrophic corneal ulcers, we covered the ulcer with an amniotic membrane 4 months after the first observation of neurotrophic keratopathy. The operation was successful, but the patient died 10 days after amniotic membrane transplantation because of complications arising from the NPC; complete healing of the neurotrophic ulcer was not achieved.

\section{Discussion}

In this report, we describe a case of neurotrophic keratopathy associated with NPC. The presence of a cavernous sinus metastasis was thought to be responsible for the severe persistent corneal epithelial defect. NPC is a squamous cell carcinoma that is relatively uncommon in most parts of the world but is more frequent in areas such as southern China, southeast Asia, and north Africa [1]. NPC is usually unresectable when diagnosed but is more responsive to radiotherapy and chemotherapy than other head and neck cancers [2]. NPC tends to undergo locoregional invasion, including extension into the cranial nerves [2].

Patients with NPC present mostly with neuro-ophthalmic symptoms including ophthalmoplegia, diplopia, and optic nerve lesions [3]. Ogunleye et al. [4] reported that 25\% of patients with NPC presented with ophthalmo-neurologic manifestations. Additionally, there are a few reports of radiation-induced optic neuropathy after radiotherapy [5, 6]. However, to our knowledge, there are no reports of corneal disease associated with NPC. In this case, 
Sato et al.: A Case of Neurotrophic Keratopathy Associated with Nasopharyngeal Carcinoma

MRI showed perineural spread extracranially along the trigeminal nerve (Fig. 3). The cornea is avascular and highly innervated by branches of the nasociliary nerve, a branch of the ophthalmic division of the trigeminal nerve. Corneal sensory nerves play an important role in the maintenance of physiological homeostasis, and impairment of these nerves can cause delayed corneal epithelium re-surfacing or corneal ulceration.

Currently, there are no well-established treatments which induce regeneration of subbasal nerves. However, several studies suggested that administration of substance-P-derived peptide, insulin-like-1 growth factor or nerve growth factor eye drops was effective in treating patients with neurotrophic keratopathy [7-9]. The development of new and more effective treatments for this refractory disease is anticipated.

\section{Statement of Ethics}

Our study protocols followed the tenets of the Declaration of Helsinki, and the patient gave his informed consent after an explanation of the possible consequences of the study. Institutional review board/ethics committee approval was obtained from Kindai University, Osaka, Japan.

\section{Disclosure Statement}

The authors have no conflicts of interest to declare.

\section{References}

1 Vokes EE, Liebowitz DN, Weichselbaum RR: Nasopharyngeal carcinoma. Lancet 1997;350:1087-1091.

-2 Guigay J, Temam S, Bourhis J, Pignon JP, Armand JP: Nasopharyngeal carcinoma and therapeutic management: the place of chemotherapy. Ann Oncol 2006;17(suppl):S304-S307.

-3 Dunmade AD, Ademola-Popoola DS: Neuro-ophthalmic manifestation of nasopharyngeal carcinoma at Ilorin: a five year review. Niger J Clin Pract 2008;11:376-378.

4 Ogunleye AO, Nwaorgu OG, Adaramola SF: Ophthalmo-neurologic manifestation of nasopharyngeal carcinoma. West Afr J Med 1999;18:106-109.

5 Zhao Z, Lan Y, Bai S, Shen J, Xiao S, Lv R, Zhang B, Tao E, Liu J: Late-onset radiation-induced optic neuropathy after radiotherapy for nasopharyngeal carcinoma. J Clin Neurosci 2013;20:702-706.

-6 Danesh-Meyer HV: Radiation-induced optic neuropathy. J Clin Neurosci 2008;15:95-100.

7 Chikama T, Fukuda K, Morishige N, Nishida T: Treatment of neurotrophic keratopathy with substanceP-derived peptide (FGLM) and insulin-like growth factor I. Lancet 1998;351:1783-1784.

8 Nishida T, Chikama T, Morishige N, Yanai R, Yamada N, Saito J: Persistent epithelial defects due to neurotrophic keratopathy treated with a substance $p$-derived peptide and insulin-like growth factor 1 . Jpn J Ophthalmol 2007;51:442-447.

9 Bonini S, Lambiase A, Rama P, Caprioglio G, Aloe L: Topical treatment with nerve growth factor for neurotrophic keratitis. Ophthalmology 2000;107:1347-1351. 


\section{Case Reports in Ophthalmology}
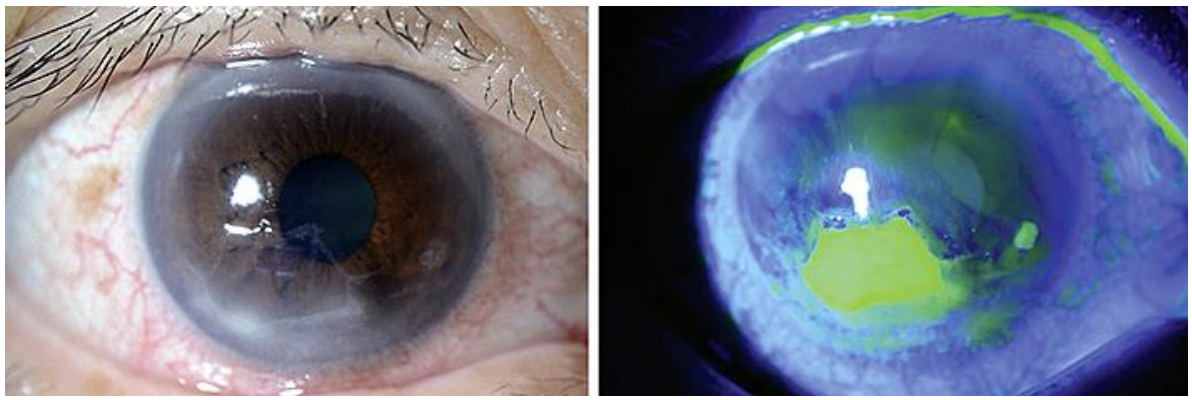

Fig. 1. Slit-lamp photographs of the right eye in a 59-year-old male diagnosed with neurotrophic keratopathy. Corneal epithelial defect and corneal stromal edema surrounding the epithelial defected area was seen in the right cornea.
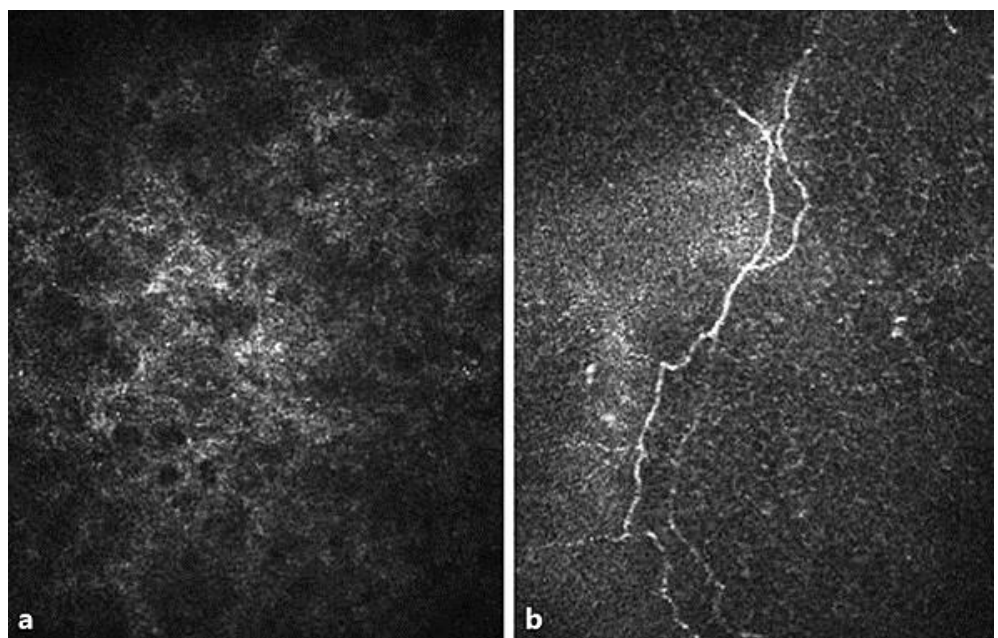

Fig. 2. Confocal microscopy images of the subbasal epithelial layer. The nerve fibers were not observed in the right eye (a) but complete nerve fibers were observed in the left eye (b). 
Case Reports in
Ophthalmology

Case Rep Ophthalmol 2018;9:114-118 DOI: $10.1159 / 000485965$

c 2018 The Author(s). Published by S. Karger AG, Basel www.karger.com/cop

Sato et al.: A Case of Neurotrophic Keratopathy Associated with Nasopharyngeal Carcinoma
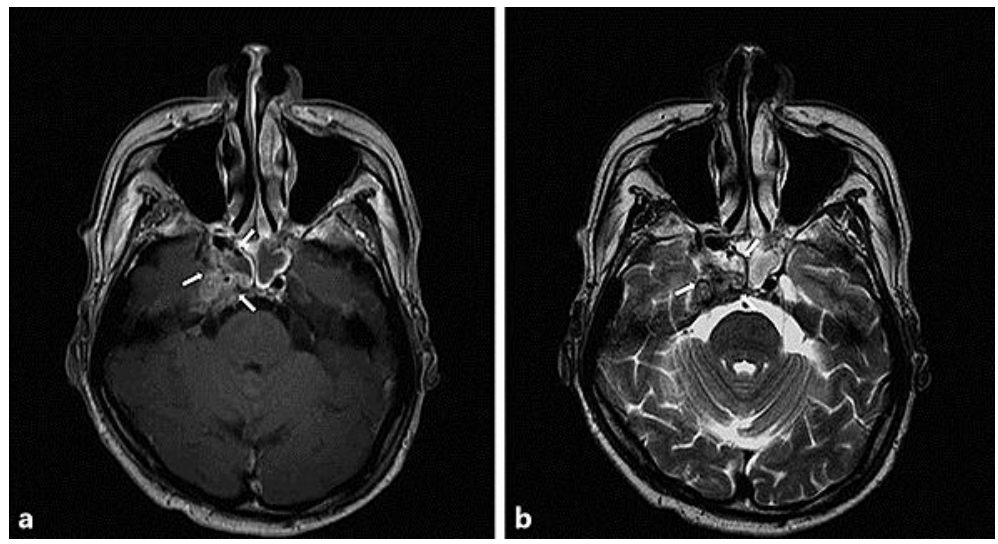

Fig. 3. a T1-weighted slices showed a mass which spread extracranially along the trigeminal nerve. b T1weighted slice showed a soft tissue tumor occupying the right Meckel's cave. The trigeminal ganglion is located within the Meckel's cave. 